\title{
Finite-difference time-domain analysis on structure-borne sound transmission characteristics of double-plate structures connected by elastically supported bars
}

\author{
Takumi Asakura* \\ Tokyo University of Science, \\ 2641, Yamazaki, Noda, 278-0022 Japan
}

(Received 10 April 2018, Accepted for publication 10 July 2018)

Keywords: Finite-difference time-domain method, Wave-based analysis, Dimension-reduced model, Floor impact sound, Bending wave PACS number: 43.40.Dx [doi:10.1250/ast.39.436]

\section{Introduction}

The vibroacoustic transmission characteristics of the floor impact sounds are greatly influenced by the vibration of the dry double floors and the suspended ceiling systems installed above/under the slabs as well as that of the floor slabs. Furthermore, the acoustic resonance phenomena in the narrow airspaces under the double floors or above the ceiling boards also affects the transmission characteristics of the sound. To predict such structure-borne sound transmission, for example, the energy-based method such as the statistical energy analysis method [1] has been applied to the vibroacoustic simulation. In contrast, the wave-based numerical analysis has also been gradually applied to such fields. Among the wave-based methods, the finite-difference time-domain method (hereafter called FDTD) has been widely applied in the fields of electromagnetics [2], seismology [3], elastodynamics [4], acoustics [5]. To simulate the vibration propagation by FDTD, the elastic wave analysis is available; however threedimensional modelling of the objects by the analysis requires higher computational costs. To reduce the costs, FDTD modelling of the plate-like and/or rod-like structures by using 2-dimensional plate elements [6] and/or 1-dimensional beam elements [7] has been investigated. By using this method, relatively costless vibroacoustic simulation of building structures is possible. The validity of the predicted results by the method has been confirmed in some case studies [8] aiming at application to real building structures; however the application of the method to the prediction of the insulation performance through double-plate structures such as the dry double floors and the suspended ceilings has not been investigated. In this paper, a basic study that aims validation of the proposed FDTD scheme on the vibration simulation of double-plate structures connected by elastically supported bars are performed. The numerical results obtained by the proposed method are validated through comparison to the results of excitation tests on acrylic models.

\section{Theory of FDTD analysis}

The proposed numerical scheme simulates planar structures like floor boards and floor slabs by using the 2dimensional plate elements, and the bar-shaped structures like

*e-mail: t_asakura@rs.tus.ac.jp the floor support legs supporting the floor boards on the slab by using the 1-dimensional bar elements. Firstly, the governing equations of vibration for plate and bar elements are described as follows. The following equations respectively describe in order, the bending wave propagation on the plate in the $x$-y plane, the in-plane wave propagation on the bar in the $x$ direction:

$$
\begin{aligned}
& D\left(1+\xi \frac{\partial}{\partial t}\right)\left(\frac{\partial^{2}}{\partial x^{2}}+\frac{\partial^{2}}{\partial y^{2}}\right)^{2} w \\
& \quad+\rho h \frac{\partial}{\partial t}\left(\mu+\frac{\partial}{\partial t}\right) w=\rho h g+p^{\mathrm{P} 1 / \mathrm{P} 2} \\
& E A \frac{\partial^{2} u}{\partial x^{2}}+\rho A \frac{\partial^{2} u}{\partial t^{2}}=\rho A g
\end{aligned}
$$

where $w$ is the displacement of the out-of-plane deformation of the plate; $u$ is the displacement of the in-plane deformation of the bar; $g$ is the gravitational acceleration; $\xi$ and $\mu$ are coefficients for modeling the damping characteristics of the bending deformation; $p^{\mathrm{p} 1}$ and $p^{\mathrm{p} 2}$ are the external forces acting on the plate; and $D$ is the flexural rigidity $(D=$ $\left.E h^{3} / 12\left(1-\gamma^{2}\right)\right)$. The other coefficients, $E, \rho, h, \gamma$, and $A$, are Young's modulus, the density, the thickness of the plate, Poisson's ratio, and the cross-sectional area of the bar, respectively. In this simulation method, the jumping of the objects is included by considering the effect of the gravity acted on the simulated object. The damping characteristics of the bending vibration using Eq. (1) are simulated by setting appropriate values of $\xi$ and $\mu$ [6] for the acrylic planar material.

The basic model of the simulated double-plate structure is shown in Fig. 1(a). The vibration transmission characteristics of the structure composed of the thick plate structure (a.1), and the thin plate structure (a.2) supported by multiple support legs (a.3) are simulated. In this model, (a.2) and (a.3) are rigidly connected whereas (a.3) are not affixed to (a.1), and the structure composed of (a.2) and (a.3) are simply put on (a.1). The vibration characteristics of the connecting part between (a.1) and (a.3), and (a.2) and (a.3) are modeled by following scheme.

Figures 1(b) and 1(c) show a discrete model composed of two plate elements and one bar element which are connected by springs. Then the plate element of Plate 1 and the bar 

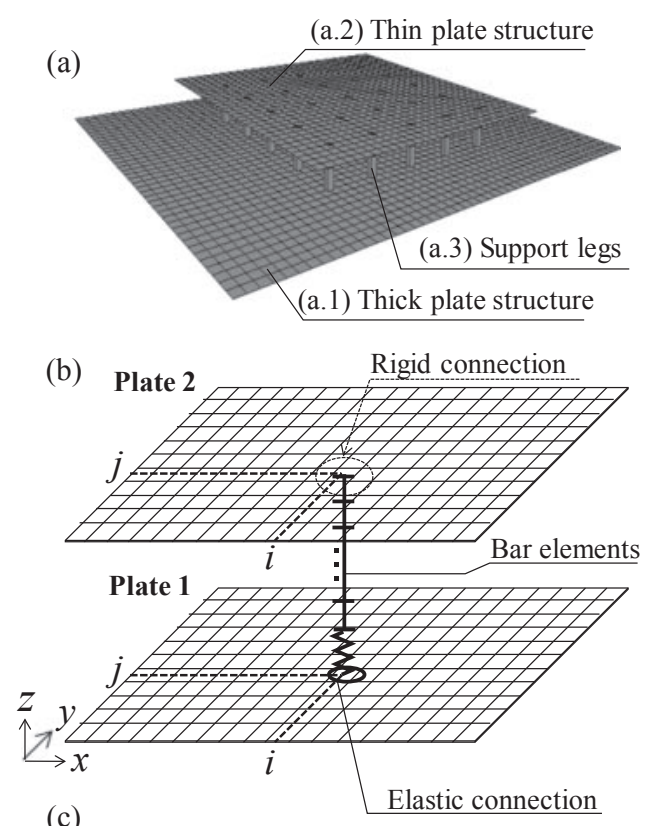

(c)

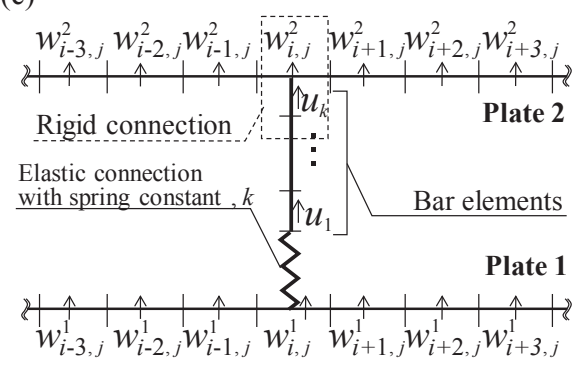

Fig. 1 Discrete model of the Beam-plate structures.

element are elastically connected by the spring whereas that on Plate 2 and the bar element are rigidly connected. Each of the vibration of the plate/bar element is calculated following the numerical scheme in [6]/[7]. In contrast, the physical relationship between the bar and plate element, and the connecting spring can be modeled by considering following continuity conditions.

Firstly, the boundary conditions on the lower/upper sides of the supporting leg as shown in Fig. 1(c) are given as Eqs. (3)/(4). As indicated in Eq. (3), the resistance force caused by the spring are acted upon the lower-side boundary of the bar. On the other hand, in Eq. (4), the displacement at the upper-side boundary of the bar equals to that of the connected element of Plate 2.

$$
\begin{aligned}
& \left.E A \frac{\partial u}{\partial x}\right|_{1}+k\left(u_{1}-w_{i, j}^{1}\right)=0, \\
& u_{k}=w_{i, j}^{2}
\end{aligned}
$$

Secondly, the $p^{\mathrm{P} 1}$ and $p^{\mathrm{P} 2}$ in the following equations which indicate the acted forces upon the Plate 1 and 2 by the spring or bar are substituted into the term of the external force $p^{\mathrm{P} 1 / \mathrm{P} 2}$ in the right side of Eq. (1).

$$
\begin{aligned}
& p^{\mathrm{P} 1}=p+k\left(w_{i, j}^{1}-u_{1}\right), \\
& p^{\mathrm{P} 2}=p+\left.E A \frac{\partial u}{\partial x}\right|_{k}
\end{aligned}
$$

Herein, the resistance force of the spring, $p^{\mathrm{P} 1}$, is acted on the element of Plate 1 whereas the axial force of the support leg, $p^{\mathrm{P} 2}$, is acted on that of Plate 2. Note that the parameter $p$ indicates the external forces other than the abovementioned force of $p^{\mathrm{P} 1 / \mathrm{P} 2}$ such as the excitation force by the impact hammer.

Lastly, the jumping of the simulated object composed of the thin plate structure (a.2) and the support legs (a.3) in Fig. 1(a) caused by reaction to such an external force as the impulsive hammering are considered as follows. The spring constant $k$ of the elastic connection parts of Fig. 1(b) is set as $k=k_{\text {sup }}$ if $u_{1}-w_{i, j}^{1} \leq 0$, whereas that is set as $k=0$ if $u_{1}-w_{i, j}^{1}>0$. Herein, the given value of $k_{\text {sup }}$ is indicated in the next chapter. The conditional update of the abovementioned parameters means that the spring works only when the spring is in compressed condition whereas that does not work in the tensile condition.

The time development of the vibration is calculated by step-by-step using the discrete equations composed of the basic equations Eqs. (1), (2), and the continuity conditions of Eqs. (3), (4), (5) and (6).

\section{Case study}

3.1. Numerical and experimental setup

An excitation test is performed on the acrylic box model of Fig. 2(a). The acrylic box has dimensions of $960 \mathrm{~mm}(\mathrm{~W}) \times$ $760 \mathrm{~mm}(\mathrm{D}) \times 1200 \mathrm{~mm}(\mathrm{H})$, and that is made of 20 -mm-thick acrylic plates which are rigidly connected with each other by welding. At the height of $590 \mathrm{~mm}$ from the under edge of the box, a partition panel is installed inside the box. In this study, two measurement conditions of Type 0 and 1 of Fig. 2(c) are investigated. Type 0 is the measurement condition to obtain the vibration of only the box whereas Type 1 is that of the box including the thinner plate with support legs. In Type 1, 5-mmthick acrylic plate with dimensions of $600 \mathrm{~mm}(\mathrm{~W}) \times 600$ $\mathrm{mm}(\mathrm{D})$ is put on the partition panel supported by 25 pieces of support legs as shown in Fig. 2(b). The support legs are arranged at $100 \mathrm{~mm}$ intervals, and they contact with the 20mm-thick partition plate through rubber pieces. The excitation points are set at $S$ shown in Figs. 2(b) and 2(c) whereas the receiving points are set at $\mathrm{R}$ (Type 0 ) or $\mathrm{R} 1$ and $\mathrm{R} 2$ (Type 1), respectively. The excitation point $\mathrm{S}$ is stuck by an impulse hammer (PCB, 086C02), and the vibration accelerances at each point are measured. Quantitatively comparing the measurement and calculation results, the accelerance is transformed into the accelerance level which is normalized by the excitation force. In the simulation, the Young's modulus, density, Poisson's ratio of acrylic plate is set as $5.6 \times 10^{9}$ $\mathrm{N} / \mathrm{m}^{2}, 1,150 \mathrm{~kg} / \mathrm{m}^{3}, 0.3$, respectively. The spring constant $k_{\text {sup }}$ of the rubber pieces having rubber hardness of $55 \mathrm{deg}$. is set as $90 \mathrm{~N} / \mathrm{mm}$. The discrete spatial and time intervals are set to be $\Delta x=\Delta y=10 \mathrm{~mm}$ and $\Delta t=1 / 48,000 \mathrm{~s}$, respectively. As the external force, a waveform simulating the acted external force of the measurement by using the Gaussian function is given. An example of the correspondence between the measured and modeled waveform of the force is shown in Fig. 3. The accelerance at each receiving point is calculated, being compared to that of the measurement. 
(a)

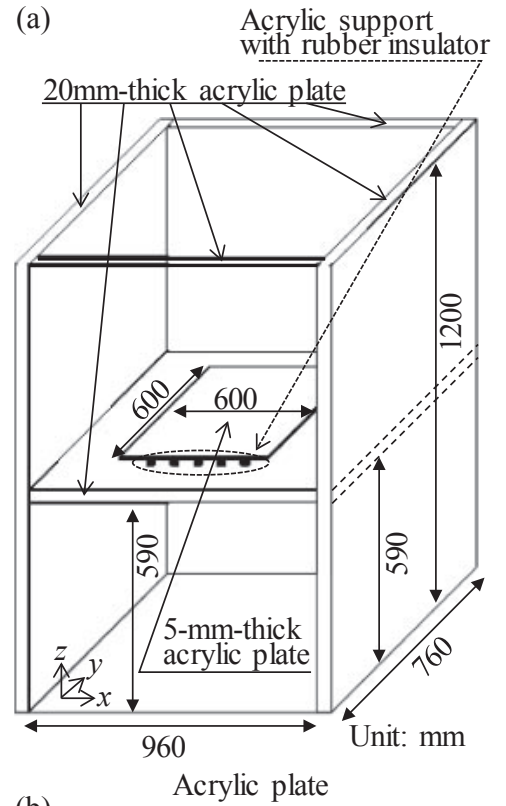

(b)

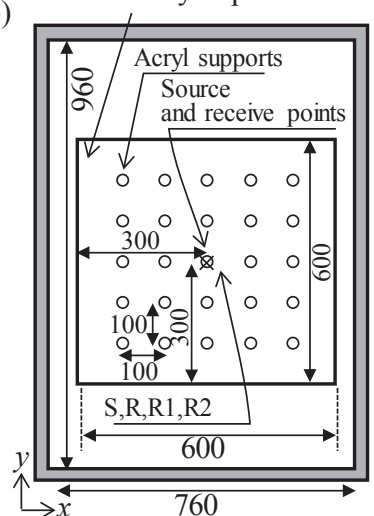

(c) Type 0 (Without thin plate)

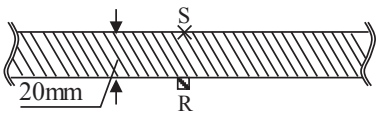

Type 1 (With thin plate)

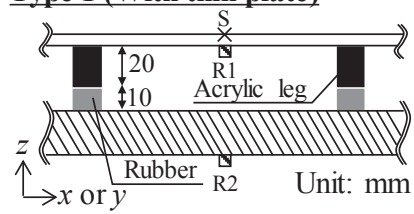

Fig. 2 Detail of the acrylic box model.

\subsection{Results and discussions}

First, the snapshots of the deformation of the simulated model obtained by the proposed FDTD calculation are shown in Fig. 4, where the gray scale represents the displacement of each plate and bar element. The amplitudes of the displacements are multiplied by 10,000. The edge part of the supported thin plate vibrates freely whereas the bending vibration of the plate is restrained by the support legs especially in the midpart.

Next, the quantitative results of the measured and calculated accelerance level are shown in Figs. 5 and 6 . Figure 5 shows the vibration characteristics of Type 0 . In the figure, the results of the measurement and calculation are in

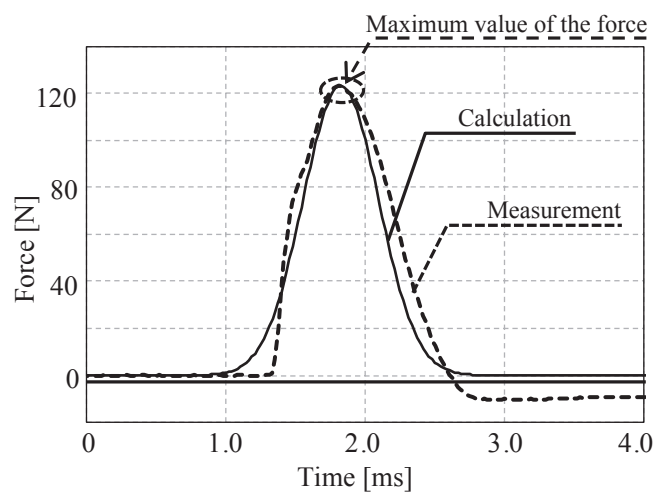

Fig. 3 Example of the input force by impulse hammer, and the simulated waveform of the acted exterior force in the FDTD calculation.

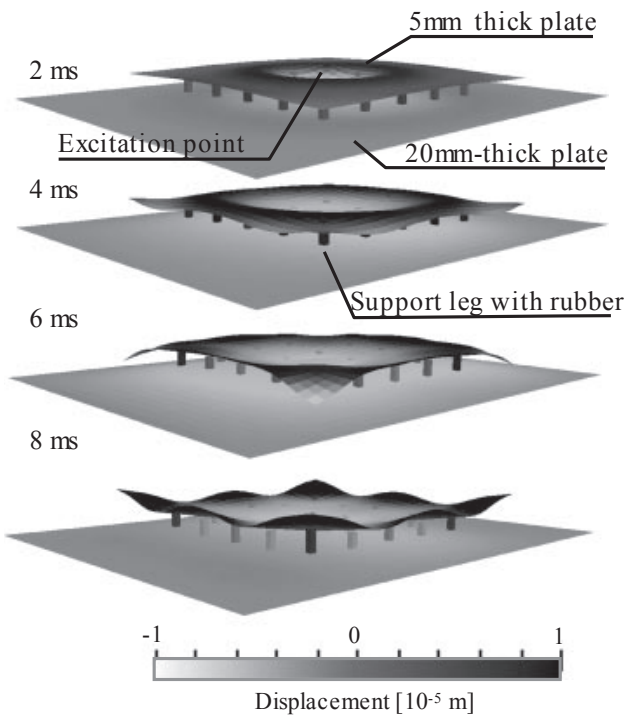

Fig. 4 Snapshots of the deformation of the simulated object by FDTD.

good agreement. Figure 6 shows the vibration characteristics of Type 1. Figures 6(a) indicates the vibration characteristics of the thin plate structure (source: S, receiver: R1), and 6(b) indicates those of the vibration transmitted to the thick plate structure (source: S, receiver: R2). The frequency characteristics of the measurement results are also well described by the calculation while the correlation between the measured and calculated results are slightly degraded compared to that of Fig. 5.

To quantitatively evaluate the similarity or difference between the calculation and measurement results, the obtained accelerance levels are converted to $1 / 3$ octave band values, and these values are comparatively plotted in Fig. 7. In the figure, the regression equations and correlation coefficients are also shown. The results of Fig. 5 have correlation coefficients $R$ of over 0.9 and gradients $\alpha$ of approximately 1.0 , and the results agree well. In the same manner, the parameters of $\alpha$ and $R$ are calculated as $\alpha=1.02, R=0.74$ for R1 of Type 1 , and $\alpha=0.92, R=0.63$ for R2 of Type 1 , 


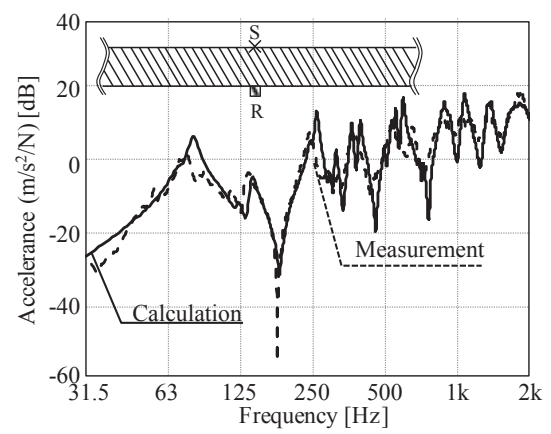

Fig. 5 Comparison of the measurement and calculation results of Type 0 .
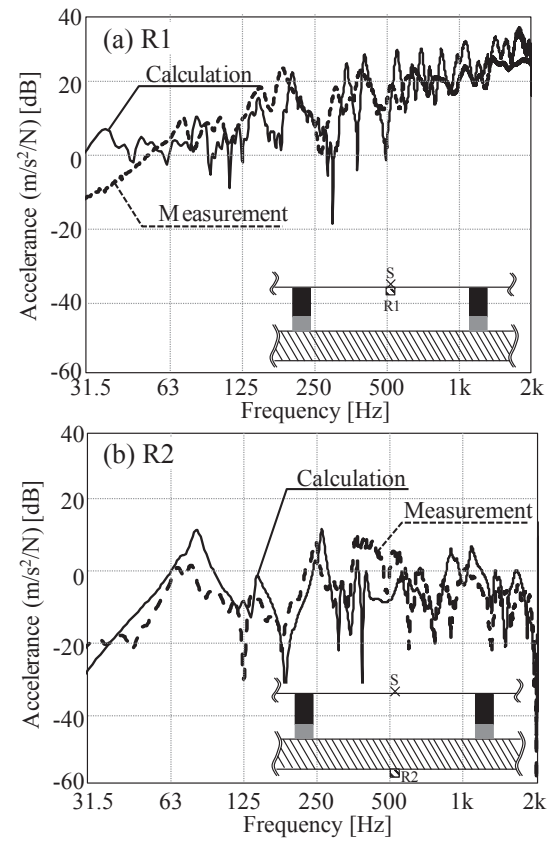

Fig. 6 Comparison of the measurement and calculation results of Type 1 .

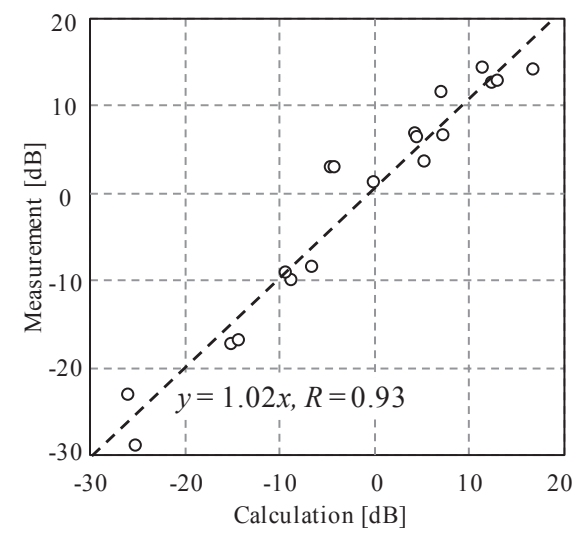

Fig. 7 Correlation of the accelerance obtained at $\mathrm{R}$ of Type 0 between the measurement and calculation results. respectively. The results of Type 1 have a little decreased correlation coefficients $R$ of 0.74 and 0.63 compared to those of Type 0 . In addition, the result obtained at R2 of Type 1 has relatively lower correlation than $\mathrm{R} 1$.

Firstly, the reason for the relatively lower correlation of Type 1 is due to more complicated structure model composed of the main structure of Type 0 and the additionally installed thin plate structure. Secondly, comparing the results of R1 and R2 in Type 1, the latter case indicates slightly lower correlation coefficient because that mainly includes the vibration transmission characteristics via the thin plate structure which is influenced by the nonlinear vibration propagation between the thick plate and the multiple support legs. However, a significant decrease of the simulation accuracy is not generally seen in the numerical results. As a result of the abovementioned agreement between the measurement and calculation, the basic validation of the proposed method is confirmed.

\section{Conclusion}

A vibration simulation method that simulates the target structure as a combination of the two-dimensional plate elements and one-dimensional bar elements is proposed. The proposed method is validated through comparison to an excitation test on an acrylic structure. The frequency-domain results obtained by the calculation agree well with the measurement. The correspondence of the measured and calculated results verified the applicability of the proposed method.

\section{Acknowledgements}

This work was supported by JSPS KAKENHI Grant Number JP17K14774.

\section{References}

[1] R. H. Lyon, Statistical Energy Analysis of Dynamical Systems (MIT Press, Cambridge, Mass., 1975).

[2] K. S. Yee, "Numerical solution of initial boundary value problems involving Maxwell's equations in isotropic media," IEEE Trans. Antennas Propag., AP-14, 302-307 (1966).

[3] J. B. Cole, "A high-accuracy realization of the Yee algorithm using non-standard finite-differences," IEEE Trans. Microw. Theory Tech., 45, 991-996 (1997).

[4] M. Toyoda and D. Takahashi, "Prediction for architectural structure-borne sound by the finite-difference time domain method," Acoust. Sci. \& Tech., 30, 265-276 (2009).

[5] D. Botteldooren, "Finite-difference time-domain simulation of low-frequency room acoustic problems," J. Acoust. Soc. Am., 98, 3302-3308 (1995).

[6] T. Asakura, T. Ishizuka, T. Miyajima, M. Toyoda and S. Sakamoto, "Finite-difference time-domain analysis of structureborne sound using a plate model based on the Kirchhoff-Love plate theory," Acoust. Sci. \& Tech., 35, 127-138 (2014).

[7] T. Asakura, T. Ishizuka, T. Miyajima, M. Toyoda and S. Sakamoto, "Vibration analysis for framed structures using the finite-difference time-domain method based on the BernoulliEuler beam theory," Acoust. Sci. \& Tech., 35, 139-149 (2014).

[8] T. Asakura, T. Ishizuka, T. Miyajima and M. Toyoda, "Numerical and experimental investigation on structure-borne sound transmission in multilayered concrete structures," $J$. Sound Vib., 413, 1-25 (2018). 